\title{
Diacronie
}

Studi di Storia Contemporanea

$N^{\circ} 27,3$ | 2016

Stato, costituzione e democrazia

\section{«La Nobiltà della Stirpe»: il sogno di un'Italia aristocratica e fascista}

\section{Lorenzo Spurio Passamonti}

\section{(2) OpenEdition}

\section{Journals}

\section{Edizione digitale}

URL: http://journals.openedition.org/diacronie/4235

DOI: 10.4000/diacronie.4235

ISSN: 2038-0925

\section{Editore}

Association culturelle Diacronie

\section{Notizia bibliografica digitale}

Lorenzo Spurio Passamonti, « «La Nobiltà della Stirpe»: il sogno di un'ltalia aristocratica e fascista », Diacronie [Online], № 27, 3 | 2016, documento 2, Messo online il 29 septembre 2016, consultato il 30 avril 2019. URL : http://journals.openedition.org/diacronie/4235; DOI : 10.4000/diacronie.4235 


\section{Diacronie}

\section{"La Nobiltà della Stirpe": il sogno di un'Italia aristocratica e fascista}

\section{Lorenzo SPURIO PASSAMONTI *}

Nell'universo dei periodici fascisti «La Nobiltà della Stirpe» rappresenta una parte della società del regime ancora poco studiata che mostra ancora di più l'eterogeneità del fascismo. Nonostante il giornale non abbia avuto fortuna di pubblico del corso della sua esistenza, dal 1931 al 1941, il suo eclettico fondatore Stefano Mario Cutelli mise tutto l'impegno possibile per ottenere il successo ed il favore di Mussolini percorrendo differenti strade e soluzioni a seconda delle occasioni. Il comitato dei redattori raccolse differenti personalità che non sempre condividevano la stessa idea di regime ma l'abilità di Cutelli fu quella di mantenere unite le differenti correnti di pensiero al servizio del sogno di un'Italia aristocratica e fascista.

\section{Introduzione}

La Nobiltà della Stirpe» nacque alla fine del 1931 e, più precisamente, grazie

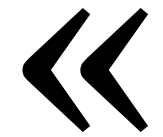
all'impulso del giovane Stefano Mario Cutelli che allora aveva solo 29 anni; come data di fondazione, venne scelto il 28 ottobre per farla coincidere con la marcia su Roma ${ }^{1}$.

La rivista chiarì, fin da subito, la sua mission: il connubio tra monarchia e fascismo, un legame saldo e indissolubile, che avrebbe portato l'Italia ai suoi più "alti destini”. Per quanto possa quindi sembrare chiaro lo scopo finale e ben delineato il percorso da seguire negli anni, il periodico dimostrò di non essere sempre coeso ma, anzi, di aver avuto al proprio interno varie correnti di pensiero che, anche se non arrivarono mai a scontrarsi, mostravano di certo quadri interpretativi molto differenti.

\footnotetext{
${ }^{1}$ La Nobiltà della Stirpe, 1, 1/1931.
} 
Non mancarono sfide con altre riviste, di cui parleremo in modo più approfondito, che dimostrano la "bellicosità" della redazione ed in particolar modo del suo direttore, sempre pronto a battersi per le sue idee.

Già negli anni in cui il periodico operava, era ben chiaro come l'alleanza SavoiaMussolini fosse per lo più un sogno, un'illusione, un accordo di comodo da offrire agli italiani, che nel tempo andò sempre peggiorando fino ad arrivare a una totale rottura ${ }^{2}$. Eppure, «La Nobiltà della Stirpe» non trattò mai di questi inasprimenti delle relazioni, indubbiamente percepiti dai senatori e dai militari vicini agli ambienti politici che affollavano il Consiglio di presidenza della rivista, ma da loro ignorati. Questo non significa che fossero disattenti o incoscienti degli sviluppi di questa difficile relazione in continuo peggioramento per via delle mire del Duce volte a sbarazzarsi della casata reale 3 - essi preferivano darne un'interpretazione molto personale, certamente distorta, ma che serviva a poter giustificare l'intero operato e continuare, così, a propugnare i propri ideali, un misto tra nostalgia e sogni di grandezza.

Prima di poter osservare con attenzione l'evoluzione del periodico è fondamentale soffermarsi sulla figura del suo fondatore/direttore, allo scopo di comprendere appieno molte delle dinamiche che animarono la vita di questa rivista.

\section{Stefano Mario Cutelli, storia di un sognatore}

Stefano Mario Cutelli nacque a Roma l'11 maggio 1902 da un'antica e nobile famiglia siciliana originaria di Milazzo; si iscrisse al PNF il primo maggio 1921 partecipando poi alla marcia su Roma4. Nel 1921 redasse un breve trattato politicofilosofico I leoni che ridono (pubblicato successivamente nel 1923), nel quale già si poteva facilmente scorgere tutta l'ammirazione per Mussolini e il fascismo dal momento che già incitava, con un anno di anticipo, a una marcia sulla capitale5. Cutelli era uno dei tanti giovani che fin dalla prima ora aveva creduto nel sogno fascista aderendo ai Fasci italiani di combattimento e mettendosi al servizio del partito attivamente e con convinzione; il suo impegno gli valse l'onore di poter recare il saluto

2 COLOMBO, Paolo, La monarchia fascista 1922-194o, Bologna, Il Mulino, 2010.

3 Ibidem.

4 DE NAPOLI, Olindo, "Come nasce una rivista giuridica antisemita. Tradizionalismo e razzismo nell'azione di Stefano Mario Cutelli», in Le carte e la storia, 12, 2/2012, pp. 98-116, p. 99.

5 LAMI, Gian Franco, Julius Evola. La Nobiltà della Stirpe (1932-1938), La Difesa della Razza (1939-1942), Roma, Fondazione Julius Evola, 2002, p. 439. 
degli universitari fascisti d'Italia in occasione dell'inaugurazione del primo anno accademico del Regime ${ }^{6}$.

Egli provò con tutto l'impegno ad affermarsi negli ambienti accademici italiani, infatti inviò la sua pubblicazione a Giovanni Gentile, Benedetto Croce e Giorgio Del Vecchio - relatore della sua tesi di laurea, con cui, successivamente, ebbe un rapporto burrascoso (dovuto principalmente all'origine ebraica del professore) - ma i suoi sforzi comunque rimasero per lo più vani7.

Dal 1924 al 1925 fu vice-segretario del GUF di Roma; pur concentrandosi sulla vita del partito, riuscì comunque a superare l'esame da procuratore. Venne inviato in Sicilia come Commissario straordinario della Confederazione generale delle corporazioni fasciste dove fondò l'istituto di Messina del Patronato Nazionale per l'assistenza sociale, divenendone direttore e, infine, costituì l'Università calabro-sicula che ebbe, però, vita breve. Per tutti questi meriti venne premiato con un'onorificenza proprio dal suo exrelatore Del Vecchio, divenuto intanto Magnifico Rettore della Sapienza ${ }^{8}$.

Negli anni successivi tentò in ogni modo di infilarsi nelle trame del mondo accademico, cercando anche di ottenere la cattedra in Dottrina del fascismo, facendo pressioni sia su Del Vecchio sia su amici del partito, ma con scarso successo. Tra il 1928 e il 1930 scrisse svariati articoli sulle più importanti riviste, tra le quali «Critica fascista» di Giuseppe Bottai, delineando inequivocabilmente la sua linea politica e cercando così di mettersi in luce, ma, ancora una volta, ciò non portò a nulla9 .

Il suo impegno politico divenne sempre più attivo: nel 1930 partecipò al Primo Congresso di studi corporativi polemizzando con Ugo Spirito, convinto sostenitore del corporativismo tanto disprezzato da Cutelli, e al Primo Convegno Nazionale della Nobiltà italiana e si persuase ancor più della necessità di una profonda riforma politicoculturale per l'Italia, riforma che avrebbe dovuto esser guidata dagli aristocratici, a braccetto con la casata reale ed il fascismo ${ }^{10}$.

Nel 1931 maturò l'idea di fondare una rivista che propugnasse questi valori e facesse da cassa di risonanza per il proseguo della rivoluzione dei fasci, al fine di creare la più sacra delle unioni: una monarchia fascista unita al ceto aristocratico. Così, nell'ottobre di quell'anno, dette vita a «La Nobiltà della Stirpe» con il sottotitolo «Roma regno italiano dei fasci di combattimento» per sottolineare ancor meglio lo spirito che animava la rivista.

\footnotetext{
${ }^{6}$ Ibidem.

7 DE NAPOLI, Olindo, «Come nasce una rivista giuridica antisemita», cit., pp. 99-100.

${ }^{8}$ LAMI, Gian Franco, op. cit., p. 439.

9 DE NAPOLI, Olindo, «Come nasce una rivista giuridica antisemita», cit., pp. 101-102.

${ }^{10}$ LAMI, Gian Franco, op. cit., p. 439.
} 
Cutelli mise l'anima affinché la rivista "spopolasse" e divenisse una voce importante nelle dinamiche del regime ma rimase, per lo più, un periodico di nicchia con uno scarsissimo seguito. Nel corso degli anni la qualità e quantità della rivista andarono sempre diminuendo finché non fu lo stesso direttore e fondatore ad abbandonarla a favore del suo nuovo progetto, "Il diritto razzista», un periodico incentrato sulle questioni giuridiche e razziali con un seguito di collaboratori anche di grande spicco ma che, suo malgrado, ebbe vita breve. "Il diritto razzista» venne data alle stampe ufficialmente nel 1940; nel 1939 era uscito il primo numero come supplemento del precedente rotocalco e nel 1942 fu costretto a chiudere i battenti per il difficile momento di guerra ${ }^{11}$.

Cutelli fu una persona certamente animata da un sincero spirito d'iniziativa e desiderio di affermazione, la sua ammirazione per il Duce era sterminata e allo stesso modo per i Savoia, in particolar modo per la figura del principe ereditario; egli credeva che il suo sogno politico potesse davvero realizzarsi, lottando contro tutti, conducendo la rivista con ardore e spregiudicatezza non mancando mai di far polemica con chiunque non gli andasse a genio ${ }^{12}$. Egli fu sicuramente un ingenuo nel credere che potesse esistere un saldo connubio tra Mussolini e i Savoia con, oltretutto, una nuova aristocrazia forte che sapesse portare avanti le sorti italiane; ciò che non vide, o non volle vedere, era il fatto che il rapporto tra i due poteri andava peggiorando e che l'aristocrazia era solo una pallida imitazione di forza e potere di quella antica che Cutelli tanto ammirava. Eppure non bisogna credere che egli non fosse attento alle dinamiche politiche che animavano l'Italia in quegli anni, anzi, dimostrò di recepire molte delle direttive mussoliniane e di intervenire con apposite rubriche o articoli su questioni importanti, volendo, alle volte, gettare acqua sul fuoco e, in altri casi, ricorrere invece alla benzina.

\section{2. «La Nobiltà della Stirpe», analisi della rivista}

La rivista, nel corso degli anni, modificò visibilmente sia l'aspetto estetico sia i contenuti, mantenendo sì l'idea di fondo, ma attraverso la mano del suo direttore e la scelta dei redattori, pose l'accento su argomenti che, a seconda delle necessità, sembravano più impellenti.

La scelta di pubblicare il primo numero ad ottobre fu l'ennesimo omaggio a Mussolini, prediligendo come data il 28 ottobre, anniversario della marcia su Roma; la

${ }^{11}$ DE NAPOLI, Olindo, «Come nasce una rivista giuridica antisemita», cit., pp. 106-110.

${ }^{12}$ Ibidem, pp. 103-104. 
copia si apriva con una dichiarazione d'intenti del periodico soffermandosi nell'importanza dell'aristocrazia italiana, della stirpe e del sangue blu' ${ }^{13}$. Gli altri articoli e le rubriche si dilungavano nel mettere in luce il valore della nobiltà italiana nella storia nazionale e il suo ruolo fondamentale nel futuro del Paese. Fin da subito, la rivista attaccava con durezza gli ideali social-comunisti, nonché democratici, sottolineando come solo i princìi aristocratici, abbinati al rigore fascista, avrebbero potuto condurre l'Italia ai più alti destini ${ }^{14}$.

Già la copertina esprimeva chiaramente i fondamenti della rivista. Su di una copertina rossa due soldati, uno in armatura medievale e uno in divisa militare contemporanea con una maschera antigas, reggevano una lastra di pietra che recava questa frase di Mussolini: «L'aristocrazia attraverso una gloriosa storia millenaria conserva pur sempre le più elette virtù della stirpe e continuerà a mantenere sotto l'egida della maestà del Re il primo posto nell'ascensione della Patria ai suoi più alti destini.»15 La frase sembrava coronare alla perfezione il sogno di Cutelli, tutte le sue idee racchiuse in una sola proposizione proveniente dall'uomo della provvidenza; in realtà, dobbiamo vedere questa frase del Duce non come un intervento programmatico, ma come uno dei tanti slogan usati nel corso degli anni per raccogliere consensi dal maggior numero possibile degli attori in scena nell'Italia del tempo ${ }^{16}$.

I due numeri successivi del 1931 continuarono su questa linea: esaltazione della monarchia e del fascismo con la spiegazione del rilievo dell'aristocrazia per il Paese, non perdendo mai occasione per attaccare bolscevichi, socialisti, democratici e chiunque non sembrasse allinearsi con il pensiero della rivista (si arrivò addirittura ad attaccare Giosuè Carducci ${ }^{17}$ ). La rivista non risparmiava toni provocanti e spericolati, volendosi dimostrare il più aggressivo possibile: «Una "civiltà", che eguaglia giuridicamente i singoli cittadini, non fa altro che degradare le classi e le famiglie superiori e sopraelevare quelle inferiori, è come un uomo che si muove a rovescio, che cammina con la testa e pensa con i piedi» ${ }^{18}$. A questi primi tre numeri parteciparono molti accademici per dare un'aura di "scientificità" alle dichiarazioni riportate.

\footnotetext{
13 «Ai Lettori», in La Nobiltà della Stirpe, 1, 1/1931.

14 La Nobiltà della Stirpe, 1, 1/1931.

15 Ibidem.

${ }^{16}$ ZUNINO, Pier Giorgio, Lìdeologia del fascismo, miti, credenze e valori nella stabilizzazione del regime, Bologna, Il Mulino, 1985, pp. 19-25

17 BRIGANTE COLONNA, Gustavo, "Continuità di gloria», in La Nobiltà della Stirpe, 1, 3/1931. Tre anni prima che la rivista fosse creata, Carducci si era espresso in modo critico sull'utilità e la funzione della Consulta Araldica perciò Brigante Colonna aveva duramente attaccato il poeta affermando che non conosceva ciò che stava criticando.

${ }^{18}$ LO SPECCHIO, «Uomini e cose allo specchio, I grandi della democrazia», in La Nobiltà della Stirpe, 1, 2/1931.
} 
I primi cinque numeri del 1932, da gennaio a maggio, seguirono tutti la stessa impostazione sia in fatto estetico sia per quanto riguardava i contenuti anche se, rispetto all'annata precedente, gli articoli offerti furono numericamente inferiori nonostante la lunghezza complessiva fosse rimasta invariata. Si continuava ad attaccare con durezza i soliti "nemici" iniziando però la lotta contro altri periodici fascisti, come ad esempio «Il secolo fascista»; questi scontri caratterizzarono tutta la vita della rivista divenendone uno dei principali caratteri distintivi. In questi numeri Cutelli, che quasi sempre firmò di proprio pugno almeno un articolo ed esercitò un rigido controllo su tutti i pezzi pubblicati dalla rivista, insisteva prepotentemente sui cavalli di battaglia che tanto gli stavano a cuore: il rinnovamento e potenziamento dei fasci attraverso un sistema gerarchico più rigido con a capo gli aristocratici, che avrebbero dovuto sostituire il PNF in tutte le sue funzioni e divenire il motore della rivoluzione fascista, il senato aristocratico ereditario, il rinnovamento delle regole araldiche, una consulta d'onore per i nobili e riconoscimenti vari per il ceto nobiliare. La rivista, come già detto, era attenta al momento politico, infatti, per la prima volta comparveroarticoli e rubriche che esaltavano l'operato della Chiesa; probabilmente, ciò fu dovuto alla pacificazione che le gerarchie cattoliche stavano operando con il regime dopo il durissimo scontro in merito all'influenza che l'Azione cattolica esercitava sul Paese, per niente gradita a Mussolini e a molti gerarchi ${ }^{19}$.

Il numero di giugno uscì, in modo inusuale, accorpato al numero di luglio. Lo stesso accadde per il numero successivo, segno questo di una carenza di contenuti che negli anni successivi si accentuò, mostrando una certa ripetitività nei temi e nei modi. La lunghezza di questo fascicolo doppio era la stessa del singolo e gli argomenti erano, nonostante la pausa di un mese, sempre identici: attacco ai social-comunisti e democratici, sbandieramento delle proprie convinzioni e proposte discutibili.

Probabilmente, la crisi non riguardava solo la ricerca di contenuti, ma anche la ricerca di fondi. Appariva, infatti, molto sospetta la nuova dicitura che compariva dietro la copertina del numero di giugno-luglio: «Questa rivista non rappresenta alcun privato interesse, ma soltanto lo spirito aristocratico: raccomandiamo quindi ad ogni nostro abbonato di procurare un abbonato nuovo» ${ }^{20}$.

Il numero per il decennale della marcia su Roma uscì in formato singolo, ma ancora una volta era piuttosto scarso di contenuti: la rivista sembrava, ad un anno dalla sua nascita, già in crisi. Infine l'ultimo numero dell'anno, anch'esso accorpato, non

19 CECI, Lucia, L’interesse superiore, il Vaticano e l'Italia di Mussolini, Roma-Bari, Laterza, 2013, pp. 152-158.

${ }^{20}$ La Nobiltà della Stirpe, 2, 6-7/1932. 
conteneva particolari note di rilievo se non che, per la prima volta, comparve la lista di tutto lo staff della rivista ${ }^{21}$, di cui parleremo in modo approfondito successivamente. Nel 1932, in ogni caso, la rivista puntava principalmente alla profusione dei propri ideali attraverso la parte più colta della redazione.

Nell'annata del 1933, venne seguita la linea già dettata, sfruttando maggiormente la parte intellettuale della redazione, battendo forte sulle medesime questioni e la rivista divenne fin troppo ripetitiva e priva di originalità. Nonostante la giovane età, «La Nobiltà della Stirpe» mostrava i primi segnali di crisi che con il tempo si sarebbero aggravati; spesso, per sopperire alla mancanza di contenuti, si ricorreva all'inserimento di recensioni letterarie, arrivando a risultare preponderanti nel numero. In questa annata si trovavano spesso articoli che trattavano in modo positivo della Chiesa e del suo ruolo equilibratore e di controllo societario; Cutelli non era un fervente cattolico anzi $^{22}$, ma l'interesse principale era quello di seguire la linea politica mussoliniana, fin tanto che non avesse leso i principi nobiliari e, se ciò fosse avvenuto, il fondatore avrebbe abilmente chiuso gli occhi, dimostrandosi un fedelissimo del Duce.

La rivista sceglieva, in modo sempre più assiduo, una linea molto dura contro i contestatori o i "nemici" degli ideali cutelliani; gli attacchi non si risparmiavano arrivando ad usare anche termini decisamente espliciti: «Il regno degli uomini di sinistra è condannato a morire e sarà probabilmente finito tra venti anni nel pieno meriggio fascista di quel secolo fascista, [...] E bisogna solo augurarsi che la espiazione dell'ubriacatura egualitaria non sia troppo dura per i poveri illusi e per la povera gente...»²3.

Infine, le difficoltà economiche sempre maggiori costrinsero la rivista a sopravvivere grazie alle donazioni di qualche personaggio facoltoso come il senatore Fabio Guidi, già membro del Consiglio di Presidenza, e con lui altri nobili che scrivevano per la rivista oppure personaggi estranei ad essa, come Guglielmo Marconi24. Gli abbonati erano pochissimi. L'ultimo numero dell'anno fece registrare un aumento del prezzo da 3 a 3,5 lire, sottolineando come il denaro ricavato non servisse tanto per creare utili quanto per portare avanti il sogno aristocratico.

L'anno successivo continuò nello stesso modo: poca originalità, stesse richieste politiche (senato ereditario, consulta d'onore, attacco ai "finti fascisti", ecc.) proseguendo negli attacchi ai periodici fascisti di sinistra, in particolare il «Secolo

\footnotetext{
${ }^{21}$ La Nobiltà della Stirpe, 2, 11-12/1932.

${ }^{22}$ DE NAPOLI, Olindo, «Come nasce una rivista giuridica antisemita», cit., p. 106.

23 CUTELLI, Stefano M., «Economia fascista e trasformismo corporativo: lettera aperta al "Secolo fascista" », in La Nobiltà della Stirpe, 3, 4/1933.

24 La Nobiltà della Stirpe, 3, 10/1933.
} 
Fascista» di Giuseppe Attilio Fanelli, nonché alla Russia bolscevica e a tutte le forze social-comuniste.

Venne istituzionalizzato il numero doppio di giugno-luglio con una dichiarazione della direzione, ma anche agosto-settembre e ottobre-novembre furono numeri doppi, lasciandoci intendere come queste non fossero scelte programmate, ma obbligate dalla mancanza di materiale da pubblicare, testimoniate dal ripetersi degli autori rispetto al periodo precedente e, probabilmente, dalla penuria economica. Verso la fine dell'anno comparvero sempre più numeri firmati da militari, iniziando così una fase ancor più intransigente della rivista. In questo anno venne più volte nominato il nazionalsocialismo e Adolf Hilter: ogni volta il giudizio fu molto duro e negativo rispecchiando come, in quei tempi, Mussolini fosse molto sospettoso del futuro alleato tedesco 25 .

L'annata 1935 segnò l'inizio di un declino inesorabile che avrebbe portato la rivista prima a faticare sempre più, pubblicando quasi esclusivamente numeri doppi, senza una precisa logica, fino ad arrivare a una preminenza dei numeri tripli ed infine all'immancabile chiusura.

In quest'anno, la rivista uscì solamente sei volte con quattro numeri doppi, uno singolo e uno addirittura triplo. Tutti furono estremamente concisi, ma si poteva notare un deciso aumento nella trattazione dei temi razziali e bio-genealogici che si muoveva su due binari: un'incomparabile superiorità della razza bianca su tutte le altre e, all'interno di essa, l'assoluta supremazia dell'aristocrazia, vista come la dominatrice fra tutte le classi e portatrice dei più alti valori civici. Questo era il tratto più caratteristico della rivista che la distingueva da tutte le altre del periodo. La linea così aggressiva ed estremista era data dalla forte presenza aristocratica conservatrice che non accettava, e non aveva accettato, di essere messa da parte negli equilibri della società, relegata in un ruolo marginale, posizione ulteriormente accentuata dalla presenza di una fazione militare dagli ideali rigidi e fortemente elitari.

Naturalmente questi articoli ebbero anche la funzione di preparare il terreno alla futura legislazione razziale e, ancor prima, all'imminente campagna etiopica; infatti, nel numero di settembre-ottobre, troviamo le motivazioni addotte dalla rivista per mettere in rilievo l'importanza di questa guerra coloniale:

[...] l'Italia praticamente sfornita di quei beni materiali, che sono la sola forza sin qui riconosciuta e obbedita, ha potuto non solo opporsi alla coalizione pluto-

25 DE FELICE, Renzo, Mussolini il duce, gli anni del consenso 1929-36, Torino, Einaudi, 1974, pp. 124-126. 
massonica-comunista in cui si era venuto sublimando il vecchio mondo demoliberale, ma ha saputo imporsi come un nuovo dinamismo politico-sociale destinato a travolgere quel mondo superato ${ }^{26}$.

In una frase sola si riuscì a mettere assieme i "nemici" del fascismo, le mancanze dell'Italia e la forza del nuovo modello politico.

Il 1936 fu certamente l'anno più disastroso per il periodico: uscirono solo quattro numeri, di cui tre doppi ed uno triplo e, senza alcuna spiegazione, non furono pubblicati i numeri di novembre e dicembre. Un vero tracollo contando, inoltre, che i fascicoli furono i più ridotti di sempre, con pochissimi articoli e rubriche: i temi toccati furono i soliti con l'unica novità rappresentata da articoli storici sull'età imperiale e agli attacchi mirati all'Etiopia. Acquistarono sempre maggior importanza gli articoli di Roberto Pavese sulla genealogia, tesi razziali, principi biologici e selettivi che mettevano in risalto l'importanza del patriziato nel mondo, unendo scienza a fantasie personali e superstizione ${ }^{27}$. Proseguendo l'andamento degli anni precedenti, i militari continuarono a prendersi sempre più spazio all'interno della rivista ${ }^{28}$.

L'annata successiva proseguì con le stesse criticità e scelte alquanto discutibili (il numero di giugno non fu pubblicato e, anche questa volta, senza motivazione), le idee sembravano essere sempre più scarse e le soluzioni sempre uguali. L'unica differenza rilevabile era la progressiva scomparsa delle richieste in merito al Senato ereditario dei Fasci (ora si richiedeva un Senato dell'Impero), la Consulta d'onore e le altre richieste che per tanto tempo erano state propugnate dalla rivista; ciò fu, probabilmente, dovuto alla rassegnazione di Cutelli, che capì che non avrebbe mai ottenuto queste riforme, per cui limitò la discussione di questi temi. In sostituzione comparvero articoli sull'Impero, attacchi ai social-comunisti, tesi razziste di varia marca, spiegazioni "scientifiche" sulla bio-genealogia ${ }^{29}$ e progressivamente ma con alcune riserve, la rivista divenne più filonazista.

Il 1938, se non altro, rese regolare l'uscita bimestrale dei numeri, ma i contenuti proposti non mostrarono alcuna svolta. La rivista tentava affannosamente di presentare l'unità di intenti e di volontà tra Corona e Duce, ma tutto ciò si presentava lontano dalla

\footnotetext{
${ }^{26}$ PAVESE, Roberto, «Dialettica delle civiltà», in La Nobiltà della Stirpe, 5, 9-10/1935.

27 PAVESE, Roberto, «Tempestività cosmica», in La Nobiltà della Stirpe, 6, 1-2/1936. Un lunghissimo articolo sulla ciclicità delle società e delle razze, ascensione del fascismo all'Olimpo degli dei. PAVESE, Roberto, «Civismo imperiale», in La Nobiltà della Stirpe, 6, 8-9/1936. Sette pagine dedicate alla spiegazione dei diritti e doveri del nuovo cittadino imperiale italiano, che avrebbero dovuto seguire i principi razziali che lo faranno sovrastare ogni altra popolazione.

${ }^{28}$ Fino al 1935 su una media di 6 articoli per numero, i militari ne firmavano un terzo, mentre dal 1936 in poi giunsero a firmarne i $3 / 5$.

29 PAVESE, Roberto, «Il mito del sangue», in La Nobiltà della Stirpe, 7, 7-8/1937.
} 
realtà all'indomani della creazione, da parte di Mussolini, della carica di Primo maresciallo dell'Impero, equiparando, almeno ideologicamente, Re e Capo del Governo: la frattura procurata non era sanabile e i rapporti tra i due poteri divennero sempre più freddi.

Nel numero di marzo-aprile cambiò il sottotitolo della rivista: «Roma Caput Mundi»30 volto a sottolineare il nuovo spirito imperiale della rivista; infatti, come già visto nelle annate precedenti, i temi egemonici divennero sempre più frequenti. A fianco di questi si continuavano a martellare i (pochi) lettori con le innumerevoli "tesi scientifiche" sull'importanza biologica-razziale del "sangue blu" e, sempre nel numero sopracitato, compariva questa nuova dicitura in copertina: «Stirpe è la parola creata dalla biologia per indicare il sangue fecondo, l'immutabile, divino microcosmo germinale che si trasmette nei secoli di padre in figlio, sangue eternamente custodito ed eternamente trasmesso»31. La linea editoriale ci appare dunque cambiata in favore di temi profondamente razziali ed estremisti, ossessionata dal volere dimostrare la forza e purezza della propria idea di società. Infine, il fascicolo annunciava l'imminente nascita del supplemento «Il diritto razzista», una rivista fondata sempre dallo stesso Cutelli che poi soppianterà «La Nobiltà della Stirpe». «Il diritto razzista» sarebbe dunque uscito col numero successivo e avrebbe portato a un aumento del prezzo della rivista a 6 lire.

Dell'annata 1939 abbiamo a disposizione solo 3 numeri, il primo uscito in versione doppia (1-2), il secondo trimestrale (3-5) ed il terzo ancora trimestrale (6-8), segno di una nuova irregolarità dovuta al maggiore impegno profuso per la rivista «Il diritto razzista» che a breve - come accennato - sarebbe divenuta indipendente da «La Nobiltà della Stirpe». La maggior parte degli articoli furono riservati a temi razziali con attacchi a ebrei, neri, "gialli", perseverando nel sostenere un principio razziale anche all'interno della società ${ }^{2}$.

È curioso vedere come, dalla metà del 1938 fino a questi ultimi numeri, divennero sempre più frequenti articoli contenenti invettive contro le donne che tentavano di emanciparsi o di dedicarsi ad attività che non erano considerate consone per loro33; sorprende vedere che questi articoli sono quasi sempre firmati proprio da una donna, che nell'ultima fase del periodico diventarono quasi preponderanti.

Le ultime due annate della rivista non sono state prese in considerazione in quanto il nuovo interesse del suo fondatore per la sua ultima creazione, «Il diritto razzista»,

${ }^{30}$ La Nobiltà della Stirpe, 8, 3-4/1938.

${ }^{31}$ Ibidem.

${ }^{2}$ FEDELE, Pietro, «La Nobiltà del sangue», in La Nobiltà della Stirpe, 9, 6-8/1939.

33 S. P. [PLONA, Stefania], "Antifemminismo della donna fascista», in La Nobiltà della Stirpe, $8,7-8 / 1938$. 
che ospitava nomi decisamente più importanti tra quelli del panorama italiano ${ }^{34}$, lo portò ad abbandonare progressivamente la prima rivista che - a dispetto del suo impegno - non aveva mai saputo affermarsi nel mondo giornalistico italiano rimanendo un piccolo periodico di nicchia.

\section{Nobili, parlamentari, militari: il Consiglio di presidenza}

Il Consiglio di presidenza fu reso pubblico per la prima volta nell'ultimo numero del 1932 e riportava i seguenti nomi: Fabio Guidi, Emanuele De Cillis, Augusto Fabbri e, naturalmente, lo stesso Cutelli35. Successivamente parteciparono al Consiglio anche Gustavo Fara, Giovanni Maresca di Serracapriola e Saverio Nasalli-Rocca ${ }^{36}$.

Di questi, la metà erano nobili: il conte Guidi, aristocratico fiorentino di lunga data37; il duca Maresca di Serracapriola, di origini napoletane che aveva ereditato svariati titoli nobiliari, aveva militato nell'esercito ed era inoltre deputato38; il conte e generale Nasalli-Rocca, anch'egli di antico lignaggio39, infine il fondatore Cutelli, che era di antica famiglia nobiliare siciliana. Questi personaggi erano accomunati da un pensiero ben preciso: la nobiltà italiana aveva imboccato una parabola discendente che necessitava quanto prima di essere bloccata ed invertita. I nobili avevano creduto fermamente nel progetto di Cutelli e sognavano come lui un'aristocrazia fascista e un fascismo nobiliare, connubio che avrebbe riportato l'Italia tra le grandi del mondo. Certamente, questi furono più realisti del fondatore della rivista e capirono molto prima che questo sogno sarebbe naufragato, fatta eccezione per il Conte Guidi che non smise mai di appoggiare e sostenere il progetto anche quando nacque «Il diritto razzista».

Il gruppo parlamentare della rivista fu utile a Cutelli per potersi affermare nelle sfere della politica ed avere contatti sia con la casata reale sia con le gerarchie fasciste.

\footnotetext{
34 DE NAPOLI, Olindo, «Come nasce una rivista giuridica antisemita», cit., pp. 111-112.

35 La Nobiltà della Stirpe, 1, 3/1931.

${ }^{36}$ La Nobiltà della Stirpe, 9, 1-2/1939.

37 Senato della Repubblica. Senatori dell'Italia fascista. Scheda Guidi Fabio, URL: <http://notes9.senato.it/web/senregno.nsf/oe10afcd14636769c1257134004b5171/9co30a5dda 9fo6a04125646foo5c8768? OpenDocument> [consultato il 5 marzo 2016].

${ }^{8} 8$ Camera dei Deputati. Portale storico. Deputato Giovanni Maresca Donnorso di Serracapriola, URL: <http://storia.camera.it/deputato/giovanni-maresca-donnorso-di-serracapriola18930111/leg-regno-XXIX\#nav > [consultato il 5 marzo 2016].

39 MISSORI, Mario, Governi, alte cariche dello Stato, Alti magistrati e Prefetti del Regno d'Italia, Roma, Ministero per i beni culturali e ambientali, 1989, p. 522 e Treccani. Dizionario biografico. Giuseppe Nasalli Rocca di Corneliano. URL:

$<$ http://www.treccani.it/enciclopedia/nasalli-rocca-di-corneliano-giuseppe_(Dizionario-

Biografico)/ > [consultato il 5 marzo 2016].
} 
Inoltre furono questi personaggi a sponsorizzare e contribuire in larga parte alla rivista nonché a trovare i contatti con importanti personaggi italiani del tempo. Fu infatti Guidi che, oltre ad essere un nobile, era un senatore, ad organizzare gli incontri di Cutelli con il Duce e con il principe ereditario di Savoia.

De Cillis, nonostante non fosse blasonato, fece stabilmente parte del Consiglio in quanto fedele fascista, convinto monarchico e che condivideva molte delle idee propugnate dalla rivista. Egli firmò solo raramente articoli, principalmente furono recensiti i suoi libri (naturalmente sempre con enormi lodi), ma il suo pensiero si poteva facilmente evincere dagli articoli firmati a nome della testata o della direzione di cui faceva parte.

Senatore era anche Gustavo Fara ${ }^{40}$, malgrado la sua principale attività fosse quella di militare, dove aveva raggiunto il grado di generale di divisione: egli aveva avuto accesso alle sale parlamentari proprio per i suoi meriti di guerra. Era un fascista convinto, che aveva partecipato alla marcia su Roma come organizzatore ${ }^{41}$, ma allo stesso tempo era un fedele monarchico, requisito fondamentale per poter far parte della rivista.

Infine, la figura del duca di Serracapriola si pose a raccordo tra le varie anime del consiglio, nonostante non fosse presente fin dalle origini; il suo ingresso ${ }^{42}$ dette equilibrio tra le varie correnti della redazione, che in quel momento si stava disgregando, grazie alla sua poliedricità come parlamentare, militare e aristocratico. Le teorie razziste "assolute", le opinioni sul nazismo, la bio-genealogia erano tutti temi che non trovavano comune accordo tra le varie personalità della rivista e che potevano causare scontri; il duca, man a mano che il periodico si orientava verso indirizzi più razzisti, decise di estraniarsi dalla direzione.

Anche la parte militare era ben rappresentata nel consiglio dai già citati Giovanni Maresca di Serracapriola, Saverio Nasalli-Rocca, Gustavo Fara e il Generale Augusto Fabbri 43 che, come sempre riportato nella rivista, era stato Capo di Stato maggiore dell'invitta terza armata ${ }^{44}$ ed era una figura da sempre fedele alla monarchia, che aveva visto nel fascismo l'occasione di far nascere una grande Italia. Non era particolarmente

\footnotetext{
${ }^{40}$ Senato della Repubblica. Senatori dell'Italia fascista. Scheda Gustavo Fara, URL: <http://notes9.senato.it/web/senregno.nsf/e38f2boo82a26247c125711400382e85/2f95969eaf b819dd4125646foo5b8be4?OpenDocument $>$ [consultato il 5 marzo 2016].

${ }^{41}$ DE FELICE, Renzo, Mussolini, il fascista, vol. I, Roma, Einaudi, 1966, pp. 343-345.

42 La Nobiltà della Stirpe, 4, 12/1934.

43 Presidenza della Repubblica. Le onorificenze. Scheda Augusto Fabbri, URL:

$<$ http://www.quirinale.it/elementi/DettaglioOnorificenze.aspx?decorato=3328 $>$ [consultato il 5 marzo 2016].

${ }_{44}$ La Nobiltà della Stirpe, 2, 11-12/1932.
} 
interessato o colpito da tutte le teorie aristocratiche che Cutelli propugnava, ma il sogno di un'Italia monarchica e fascista era troppo allettante per rinunciarvi.

Tutte queste figure hanno tratti comuni che, all'inizio del progetto e fino circa al 1936, seguirono un'unità di intenti, poi, con il radicalizzarsi delle idee estremiste di Cutelli e della frangia più intransigente della rivista, Fabbri, Nasalli-Rocca, Maresca di Serracapriola e De Cillis, decisero di farsi da parte e collaborare solo saltuariamente, infastiditi da queste idee.

\section{Gli intellettuali: Pellizzi ed Evola}

Nel corso degli anni vi furono svariati intellettuali, accademici e non, a partecipare alla vita editoriale della rivista dando il proprio personale apporto, non allineandosi in modo supino alle idee di Cutelli, ma esprimendo principi indipendenti che, alcune volte, non raccolsero il favore della direzione. Questi operarono principalmente nei primi tre/quattro anni e, successivamente fino al 1941, anno della cessazione, furono sempre di meno coloro che vollero partecipare, rimanendo attivi solo quelli che erano più fermamente convinti degli ideali razzisti e, in seconda battuta, dei principi biogenealogici di superiorità dell'aristocrazia.

Il gruppo era molto eterogeneo, sia per provenienza sociale, sia per formazione; tra di essi si potevano annoverare: professori universitari, dottori, professori, nobili, avvocati e ancora senatori45. Questi non erano uniti: tra alcune anime di questo gruppo esistevano dissidi, antipatie e scontri ideologici che avrebbero potuto minare l'integrità della rivista ${ }^{46}$. L'abilità nel tenere salda questa formazione fu dell'eccentrico direttore, che ridusse al minimo lo spazio di libertà di questi autori all'interno del periodico, egemonizzando la scelta degli articoli e delle rubriche, aggiustandole in modo tale da renderle il meno contrastanti possibile con le idee della rivista e degli altri intellettuali del comitato di redattori.

Non ci soffermeremo su tutti coloro che scrissero per la rivista ma solamente sulle figure più importanti che espressero un pensiero originale e non sempre allineato con quello del fondatore, tralasciando, coloro che seguirono pedissequamente la linea impostata.

45 La rivista contava un totale di 37 effettivi tra redattori, collaboratori e consiglio di presidenza, di questi 6 erano militari, 7 senatori o parlamentari, 11 tra avvocati e dottori, 9 i professori universitari e 4 impiegati comuni.

46 PERFETTI, Francesco, Fascismo monarchico, i paladini della monarchia assoluta fra integralismo e dissidenza, Roma, Bonacci, 1988, p. 121. 
Stefano Mario Cutelli fin dalla nascita del suo progetto sognava un'internazionale fascista di carattere monarchico che potesse espandersi in tutto il territorio europeo. L'uomo che egli aveva individuato per contribuire a questo progetto era Camillo Pellizzi, uno dei tanti giovani ammaliato dal sogno del fascismo, che si era iscritto al partito il 19 giugno 1921 e, dopo essersi formato negli ambienti accademici italiani e inglesi, si trasferì a Londra dove fondò il Fascio londinese e divenne poi professore di lingua italiana presso la locale università47. Furono proprio queste caratteristiche a far cadere l'attenzione di Cutelli su Pellizzi che, sia nell'ambiente italiano sia in quello inglese, era molto affermato, malgrado questo studioso avesse idee anche molto distanti da lui. Ad esempio l'amicizia che lo legava a Ugo Spirito e Giuseppe Attilio Fanelli48, entrambi avversari politici di Cutelli, dimostravano l'elasticità mentale del direttore e il suo interesse a collaborare con più movimenti del fascismo. Pellizzi non partecipò mai in prima linea alla rivista, ma alcuni suoi articoli e pensieri, ritagliati ad hoc, furono sfruttati per mettere in buona luce le idee che stavano a cuore al direttore: l’importanza del fascismo e la predominanza dell'aristocrazia.

Proprio per questo venne riportata parte di un articolo di Pellizzi, per la prima volta, nel terzo numero del 193149: si discuteva della mancanza di una forte élite dirigente nel neonato Stato fascista. La soluzione che egli proponeva era quella di fare affidamento sulle forze che da sempre si erano distinte nell'organizzazione del potere: l'aristocrazia.

Ciò porterebbe a supporre un'uniformità di pensiero tra Pellizzi e Cutelli, ma in realtà vi erano tra i due profonde e insanabili differenze. Il fondatore de «La Nobiltà della Stirpe» era un estimatore dell'aristocrazia, del fascismo di destra conservatore e della monarchia e ripudiava tutto il resto, che doveva essere sottomesso, oppure, eliminato, in primis la piccola borghesia, i social-comunisti e i proletari. Pellizzi non era né un nobile, né un particolare estimatore dell'aristocrazia italiana, ma riconosceva in questa delle capacità che avrebbero potuto aiutare il paese. Egli era ben lontano dalle idee di Cutelli, in particolar modo, per quanto riguardava la borghesia, che Pellizzi vedeva come una forza promotrice di innovamento e vera custode della rivoluzione fascista. Questo creava una frattura insanabile tra i due che causò, infatti, una prematura interruzione della loro collaborazione.

47 MISSORI, Mario, Gerarchie e statuti del PNF, Gran Consiglio, Direttorio nazionale, federazioni provinciali: quadri e biografie, Roma, Bonacci, 1986, p. 255.

48 BRESCHI, Danilo, LONGO, Gisella, Camillo Pellizzi, la ricerca delle élites tra politica e sociologia, Soveria Mannelli, Rubettino, 2003, pp. 104-105.

49 PELLIZZI, Camillo, «Sulla esigenza di un patriziato del regime fascista», in La Nobiltà della Stirpe, 1, 3/1931. 
Vennero pubblicati ancora altri due articoli, uno nel febbraio del $1932^{5}$ ed un altro nel maggio dello stesso anno ${ }^{1}$ in cui, ogni volta, Cutelli "aggiustava" le notizie in maniera tale da adattarle al pensiero della rivista, aggiungendo sempre una nota in fondo al pezzo che giustificava le differenze di pensiero che correvano tra i due, in modi, suo malgrado, molto maldestri tanto che, nel numero di maggio, Pellizzi inviò una lettera aperta alla rivista in cui criticava il direttore per aver distorto le sue idee. Cutelli non poté far altro che abbozzare una difesa goffa che cercò di smussare gli angoli del suo intervento, ma ormai l'errore era stato compiuto e non poteva essere sanato. Non ci furono altre collaborazioni tra le due parti, eccetto un breve articolo riportato nel numero di dicembre del 1934.

Pellizzi, nel 1949, pubblicò Una rivoluzione mancata, un testo in cui stilava un bilancio dell'esperienza fascista volendo spiegare, a suo parere, le ragioni che avevano portato al fallimento di questo progetto. Egli continuò a sostenere che il fascismo sarebbe potuto essere la forza che avrebbe potuto risollevare l'Italia, ma il fallimento si era compiuto nel momento in cui questi avevano abbandonato «il vigore morale e il rigore intellettuale» 52 facendo decadere tutto il sistema. Pellizzi, nonostante il periodo di caccia al fascista, non ebbe paura di continuare a sostenere il fascismo nella sua accezione corporativista. Ciò che a noi interessa è vedere come in questo volume egli non abbia mai citato una volta Cutelli e le sue idee a ulteriore dimostrazione della distanza di Pellizzi dal fondatore della rivista53.

L'altro grande intellettuale che partecipò alla vita del periodico, in modo oltretutto molto più attivo e partecipe rispetto Pellizzi, fu Julius Evola, una poliedrica figura di studioso del panorama italiano che, a momenti alterni, apprezzò e criticò i due grandi regimi di destra del tempo. Mussolini apprezzava molto Evola per il suo amore per la romanità 54 nonché per il suo spiritualismo, in compenso, alcune delle sfaccettature di questo personaggio, come le rigidi suddivisioni societarie in cui credeva, non piacquero al Duce, che preferì lasciarlo sempre in una posizione marginale nel panorama accademico italiano.

Cutelli, come Mussolini, apprezzava molte delle idee di Evola e non mancò di esaltarlo più volte in rubriche e articoli arrivando a dire di lui: «J. Evola: il più estremo

50 PELLIZZI, Camillo, «Aristocrazia, fascio famigliare, ordine senatorio», in La Nobiltà della Stirpe, 2, 2/1932.

51 PELLIZZI, Camillo, "Camera elettiva e senato ereditario», in La Nobiltà della Stirpe, 2, 5/1932.

52 SALVATI, Mariuccia, Introduzione, in PELLIZZI, Camillo, Una rivoluzione mancata, Bologna, Il Mulino, 2009, p. 23.

53 Ibidem, pp. 21-25.

54 GENTILE, Emilio, Il culto del littorio, la sacralizzazione della politica nell'Italia fascista, Roma-Bari, Laterza, 2009. 
filosofo dell'estrema destra»55, vedendo in lui quell'uomo che sarebbe potuto diventare il più grande sostenitore del progetto aristocratico conservatore, permeato di un sano razzismo e fedeltà alla Corona. In realtà le posizioni dei due erano molto differenti, ma il direttore preferiva chiudere gli occhi sulle differenze, anche se importanti, e continuare a sponsorizzare i suoi lavori pubblicando, tra il 1932 e il 1934, moltissimi suoi articoli56.

Evola condivideva alcune delle idee di fondo di Cutelli come, ad esempio, la rivitalizzazione dell'aristocrazia italiana combattente contro le nuove forze socialdemocratiche che rischiavano di distruggere le solide base gettate dal mondo moderno ma, allo stesso modo, molti dei pensieri e tesi sostenute dalla rivista erano profondamente contrarie al pensiero di Evola, che in primis respingeva l'idea di un razzismo biologico di marca aristocratica, caposaldo, invece, di Cutelli57. Evola sosteneva altresì un razzismo basato sullo spiritualismo che poteva comprendere o meno gli aristocratici nei quali, in ogni caso, riponeva le migliori speranze58.

Come Pellizzi, Evola era amico di rappresentanti delle varie correnti del fascismo tra cui Fanelli, uno dei più disprezzati "nemici" del fondatore de «La Nobilita della Stirpe», con cui aveva avuto saltuarie collaborazioni e, pur non sempre in accordo tra di loro, esisteva un rapporto di fiducia e apprezzamento, sul quale Cutelli preferì sorvolare 59 .

Infine, Evola decise di allontanarsi volontariamente dalla rivista, stanco delle manipolazioni dei suoi articoli che dovevano sempre apparire perfettamente in linea con il pensiero del direttore anche se, spesso, queste non potevano naturalmente coincidere e dovevano, per forza di cose, subire aggiustamenti e mutazioni con interpretazioni, a dir poco, fantasiose. Inoltre, il rapporto tra i due si era fatto sempre più teso per via del progressivo radicalizzarsi di Cutelli, che portò Evola ad allontanarsi progressivamente fino al completo distacco ${ }^{60}$. Malgrado ciò il nome di Evola non scomparve mai dalla lista dei redattori, come anche quello di Pellizzi, per via della loro importanza nazionale e internazionale.

Possiamo dunque affermare come il rapporto che «La Nobiltà della Stirpe» mantenne con i suoi intellettuali fu piuttosto burrascoso. Progressivamente coloro che appartenevano al mondo accademico decisero di farsi da parte volontariamente, non

55 CUTELLI, Stefano M., «Rivolta contro il mondo moderno», in La Nobiltà della Stirpe, 4, 3/1934.

${ }^{5}$ LAMI, Gian Franco, Julius Evola, cit., pp. 19-24.

57 DE FELICE, Renzo, Storia degli ebrei italiani sotto il fascismo, Torino, Einaudi, 1961, p. 447.

${ }^{8}$ Ibidem.

59 PERFETTI, Francesco, Fascismo monarchico, cit., pp. 361-363.

${ }^{60}$ LAMI, Gian Franco, Julius Evola, cit., p. 33. 
riconoscendosi più nelle idee propugnate. Coloro che rimasero a fianco della rivista per tutta la sua durata, ad esempio Roberto Pavese, erano degli estremisti di destra e convinti razzisti. Non sarebbe potuta andare in altro modo, visto che il fascismo, almeno in alcune sue parti, si può definire razzista e totalitario non solo nell'ultima fase, quando raggiunse l'apice ${ }^{61}$; la rivista seguì di pari passo questo andamento e - pur continuando a sostenere l'importanza dell'aristocrazia - questa tematica pian piano si trasferì in secondo piano rispetto alla questione razzista.

\section{Collaboratori, vari ed occasionali}

È sorprendente vedere come Cutelli immise nella rivista quante più personalità possibili, di estrazione diversa, di grado sociale assai differente e con idee anche dissonanti tra loro; ciò che parve preoccupare principalmente al fondatore era riunire il maggior numero di soggetti, smussandone naturalmente le idee, per poter vantare un comitato di redattori più vasto e aperto, che sapesse quindi confrontarsi con più correnti di pensiero ${ }^{62} \mathrm{e}$ ottenere, in aggiunta, molta pubblicità.

Come abbiamo visto precedentemente, militari, nobili e parlamentari erano la parte più consistente della redazione e del Consiglio di presidenza, ma Cutelli decise di coinvolgere anche persone che non avevano estrazione nobiliare e nemmeno una posizione sociale di rilievo come Ernesto Baranger, Stefano Carducci e Stefania Plona.

I tre avevano un'origine umile e in netto contrasto con il livello sociale degli appartenenti alla rivista ma Cutelli, visto il calo dei collaboratori della rivista e la mancanza di materiale da pubblicare, tentò una via nuova aprendo la strada ai tre e offrendo loro spazi all'interno della rivista. Essi non scrissero mai gli articoli principali - come l'editoriale o gli articoli più lunghi -, ma dettero il loro contributo in modo significativo volendo mostrare un ceto popolare che approvava in pieno le scelte dei ceti superiori, felice di essere guidato nella società. Il tutto ci appare surreale, in particolar modo quando vediamo scrivere Stefania Plona contro il modernismo delle donne affermando che esse avrebbero dovuto rimanere in casa e occuparsi esclusivamente della famiglia.

\footnotetext{
${ }^{61}$ GENTILE, Emilio, Il culto del littorio, la sacralizzazione della politica nell'Italia fascista, Roma, Laterza, 1988, p. 174.

${ }^{62}$ Cosa assolutamente falsa in quanto Cutelli dominava quasi totalmente gli articoli e le rubriche e, quando non era egli stesso a firmare un articolo, preferiva affidarsi a membri della rivista di cui si fidava ciecamente; infatti molti nomi sono nel corso degli anni ricorrevano, mentre altri comparivano saltuariamente.
} 
Nell'ultima fase di vita del periodico pochi degli storici collaboratori continuarono a redigere articoli e rubriche poiché, nel momento in cui Cutelli decise di radicalizzare profondamente il suo pensiero, la maggior parte di essi abbandonò la rivista. Il direttore fu, dunque, costretto a cercare nuove soluzioni per poter mantenere un pubblico, anche se minimo, continuando il sogno di un'aristocrazia fascista nonostante il paradosso di coinvolgere in questo progetto coloro che in realtà ne avrebbero avuto un risultato negativo per la loro condizione. Ancora una volta il direttore preferì non vedere questa contraddizione, come già fatto in passato per molte altre situazioni analoghe: ciò che più lo interessava era potersi affermare e sostenere le proprie idee. Tale missione, che non gli era riuscita con «La Nobiltà della Stirpe», lo portò, quindi, a cercar fortuna attraverso la sua nuova rivista, nata, come detto, quale appendice del suo vecchio periodico, «Il diritto razzista». Questa, infatti, poteva vantare un comitato di redattori decisamente più prestigioso ${ }^{63}$, appoggi politici più forti ed era foriera di un tema, caldo e maggiormente approvato, che aveva un pubblico decisamente più vasto.

I tre non produssero materiale particolarmente interessante, limitandosi per lo più alla produzione di poetica e a qualche pubblicazione che sosteneva le tematiche care al direttore non distinguendosi, perciò, dal resto della redazione. In ogni caso, è comunque interessante osservare la partecipazione di italiani di "classe sociale inferiore" al progetto di una rivista aristocratica.

I collaboratori che non abbiamo citato, come detto precedentemente, appartengono alle classi sociali che avevano appoggiato la rivista. Tutti subirono il controllo del direttore e non ebbero particolari spazi di manovra producendo quindi articoli e rubriche vicini al pensiero di Cutelli che, quindi, non aggiunsero niente di originale ai temi già esposti.

\section{Bilancio di un fallimento}

La rivista fu un fallimento, ma va riconosciuto un percorso e un impegno non indifferente che non fu tanto frutto del caso e dell'improvvisazione, quanto di un progetto ben delineato che, suo malgrado, non riuscì mai ad affermarsi.

In primis Cutelli distorceva la realtà vedendo un'aristocrazia che poteva rialzarsi e tornare a essere protagonista della vita politica ed economica del paese riaffermando,

63 Tra i tanti che parteciparono alla rivista possiamo citare: Santi Romano, Rende Domenico (Presidente di Sezione della Corte di Cassazione), il ministro Pietro Fedele, Savorgnan Franco (Presidente dell'Istituto centrale di Statistica Silverio Leicht (Presidente della facoltà di Giurisprudenza romana), Adolfo Giaquinto (avvocato generale dello Stato) e molti altri importanti nomi dell'Italia fascisti sostenitori delle tesi razziste. 
come nel passato, il ruolo egemone nella società. La nobiltà di tutta Europa aveva subito un fortissimo contraccolpo dalla Grande guerra, dai nuovi movimenti di massa nonché da politiche economiche che avevano colpito fortemente i patrimoni familiari ${ }^{64}$ : questo ceto era, dunque, avviato ad un lento ma inesorabile declino. La nobiltà italiana non faceva eccezione a questo processo di decadimento. Secondo un censimento del 1922, era arrivata ad una quota della popolazione inferiore al 2\%65 mantenendo, sì, alcuni ruoli di spicco, ma perdendo ogni incisività nelle scelte decisionali della Nazione.

In secondo luogo, il fondatore della rivista credeva in un fascismo che appoggiava e sosteneva apertamente l'aristocrazia italiana ed, effettivamente, il Duce aveva fatto pubbliche dichiarazioni in cui sembrava supportare la causa della nobiltà, ma altro non era che semplice propaganda per raccogliere consensi ${ }^{66}$. Infatti, se è vero che il regime favorì l'ascesa di nuovi nobili con concessioni di titoli e promulgò alcune norme in favore di questo ceto, come ad esempio il riconoscimento della nobiltà papalina - che lo stato liberale aveva negato - oltre ad altri piccoli interventi, Mussolini non volle esporsi ulteriormente e, già dalla fine degli anni Venti, era maggiormente interessato ad altri progetti piuttosto che a guadagnare il favore dell'aristocrazia ${ }^{67}$.

Infine, va osservato il ruolo della monarchia in queste vicende considerando che, assieme ai primi due, era il terzo caposaldo di Cutelli. Teoricamente il trinomio fascismo-monarchia-aristocrazia doveva essere la base della nuova Italia imperialistica e possente di fronte al mondo. La Corona non aveva preso una posizione precisa in merito alla nobiltà lasciando che Mussolini potesse appropriarsi delle prerogative di controllo su questo ceto; attraverso il controllo totalitario dello Stato avocò a sé anche la prerogativa di nominare nuovi nobili e controllare la Consulta Araldica68. Ciò che Cutelli tentò maldestramente di nascondere attraverso gloriosi articoli era quanto il rapporto tra monarchia e fascismo s'incrinasse ogni giorno di più, cercando, quindi, di rappresentare un sacro legame forte della stessa unità d’intenti: nulla di più lontano dalla realtà.

Queste tre prerogative, che il fondatore de «La Nobiltà della Stirpe» riteneva esistenti e, naturalmente, essenziali per la realizzazione del suo progetto erano pressoché inesistenti: l'aristocrazia italiana era debole, disunita e senza un progetto comune; il fascismo era quasi totalmente disinteressato delle sorti del ceto nobiliare,

\footnotetext{
64 JOCTEAU, Gian Carlo, I nobili del fascismo, in «Studi Storici», 45, 3/2004, pp. 677-726, pp. 677-678.

65 Ibidem, pp. 679-682.

${ }^{66}$ Ibidem, pp. 683-688.

67 Ibidem.

68 Ibidem, pp. 695-696.
} 
preoccupato di questioni molto più urgenti e importanti; la monarchia, infine, non li appoggiava ed era ben lontana da un felice rapporto con il regime ma anzi esisteva un abisso incolmabile.

Queste motivazioni portarono al fallimento degli obiettivi della rivista e di quel circolo che credeva fortemente nel progetto ma che, ad una valutazione più attenta ed equilibrata, avrebbe dovuto comprendere sin dal principio l'impossibilità di tale opera.

Non sappiamo con certezza se Cutelli si rendesse conto della mancanza di elementi fondamentali per riuscire nei suoi intenti o se volesse ignorare i fattori ambientali e proseguire per la propria strada, tesi che appare più plausibile, ma una cosa è certa: egli mise tutto l'impegno possibile e tentò tutte le strade per accrescere le possibilità di successo. Il tentativo maldestro di conciliare gli ideali di molti differenti intellettuali è un esempio chiarissimo dello sforzo che compì per ottenere il consenso della rivista $\mathrm{e}$, quando questi decisero di voltargli le spalle, non demorse, anzi, decise di percorrere nuove strade puntando sui militari presenti in redazione fino ad arrivare a personaggi di ceti sociali inferiori.

Cutelli era uno di quei giovani rimasti affascinati dai miti che la propaganda fascista propugnava ogni giorno fin dal momento della sua nascita: la venerazione di Roma e della romanità come base della stirpe italiana e dei valori italiani ${ }^{69}$, il culto civico del fascismo con caratteri di religiosità che aveva avuto il merito di mettere a tacere i "Rossi"7o, nonché l'esaltazione costante dell’importanza di Mussolini per le sorti della nazione. Solo ricordando gli elementi costitutivi del momento possiamo capire come si fosse sviluppata l'identità di questa personalità e come poi, partendo da queste "verità", abbia sviluppato il suo pensiero sull'aristocrazia fascista. Non va dimenticato, infatti, che Cutelli proveniva da un'antica famiglia nobiliare di origine siciliane il che, certamente, contribuì a creare in lui un ulteriore mito, quasi un dogma: la potenza dell'aristocrazia e la sua importanza nella storia, che egli voleva trasferire nel suo tempo.

Alla fine anche Cutelli dovette fare i conti con la realtà. Il suo progressivo abbandono della rivista si compì in più fasi: prima smise di trattare con assiduità i temi riguardanti la nobiltà, cessando di fare le sue assidue richieste di rinnovamento della società in chiave aristocratica; poi tralasciò sempre di più la cura della rivista, che progressivamente si svuotò dai contenuti, infine si adoperò per creare un nuovo periodico «Il diritto razzista» che non trattava più i temi che tanto aveva avuto a cuore negli anni precedenti. Il canto del cigno arrivò con l'8 settembre 1943: Mussolini,

69 GENTILE, Emilio, Il culto del littorio, cit., pp. 129-135.

70 Ibidem, pp. 96-97. 
liberato dai nazisti, fondò la Repubblica sociale italiana e gli italiani che si trovarono all'interno dei suoi confini furono costretti a decidere se aderirvi o meno, anche se spesso non fu loro lasciata molta scelta ${ }^{71}$. Cutelli aderì spontaneamente alla RSI e questo dimostrò che egli teneva di più al Duce ed al suo mito che alla monarchia, che si era schierata con gli Alleati fuggendo al Sud72. Avrebbe potuto svincolarsi da questa situazione, ma aderì perché convinto della causa del Duce nonostante anche la maggioranza della nobiltà italiana, oltre alla Corona, avesse abbandonato da tempo il fascismo e Mussolini. Dopo la definitiva caduta del fascismo Cutelli non subì processi epurativi in quanto non gli fu riconosciuto un ruolo importante ed attivo, né come collaborazionista, né come fascista della prima ora73.

Il suo sogno era miseramente fallito e man mano che l'aveva visto sgretolarsi si era aggrappato alle convinzioni che gli erano rimaste arrivando, infine, a lottare per l'ultima cosa che gli era rimasta. Il fascismo di Salò, nonostante fosse solo una pallida imitazione del regime ${ }^{74}$, non più in grado di esercitare un potere che non fosse mera violenza, suscitò l'approvazione di Cutelli e il suo sostegno, malgrado apparisse evidente la sua prossima sconfitta; egli, ancora una volta, aveva preferito chiudere gli occhi piuttosto che confrontarsi con la realtà dei fatti. Dopo la guerra trascorse una vita ritirata non impegnandosi più in alcun modo in campo politico: di lui era rimasto solo il sognatore senza più il sogno da perseguire.

\footnotetext{
${ }^{71}$ DE FELICE, Renzo, Mussolini l'alleato, vol. II, Roma, Einaudi, 1990.

${ }_{72}$ LAMI, Gian Franco, Julius Evola, cit., p. 440.

73 Ibidem.

74 FRANZINELLI, Mimmo, Le stragi nascoste, l'armadio della vergogna: impunità e rimozione dei crimini di guerra nazifascisti 1943-2001, Milano, Mondadori, 2002, pp. 61-74.
} 


\section{* L'autore}

Lorenzo Spurio Passamonti (Umbertide 1993) si è laureato in Beni e attività culturali presso l'Università di Perugia nel novembre 2015 con il punteggio di 110. Attualmente è iscritto al corso di laurea specialistica in Scienze storiche presso l'Università di Bologna (storia contemporanea); si occupa, in particolare del periodo dei totalitarismi e del secondo dopoguerra italiano ed europeo. Lavora per la pro loco di Corciano (PG).

URL: < http://www.studistorici.com/progett/autori/\#SpurioPassamonti >

\section{Per citare questo articolo:}

SPURIO PASSAMONTI, Lorenzo, "“La Nobiltà della Stirpe" il sogno di un'Italia aristocratica e fascista», Diacronie. Studi di Storia Contemporanea : Stato, costituzione e democrazia, 29/09/2016,

URL:< http://www.studistorici.com/2016/09/29/spurio-passamonti_numero_27/ >

Diacronie Studi di Storia Contemporanea $\vartheta$ www.diacronie.it

Risorsa digitale indipendente a carattere storiografico. Uscita trimestrale. redazione.diacronie@hotmail.it

Comitato di redazione: Jacopo Bassi - Luca Bufarale - Elisa Grandi - Antonio César Moreno Cantano - Deborah Paci - Fausto Pietrancosta - Alessandro Salvador - Matteo Tomasoni - Luca Zuccolo

Diritti: gli articoli di Diacronie. Studi di Storia Contemporanea sono pubblicati sotto licenza Creative Commons 3.0. Possono essere riprodotti e modificati a patto di indicare eventuali modifiche dei contenuti, di riconoscere la paternità dell'opera e di condividerla allo stesso modo. La citazione di estratti è comunque sempre autorizzata, nei limiti previsti dalla legge. 